OPEN ACCESS

Edited by:

Jianguang Ji,

Lund University, Sweden

Reviewed by:

Wang Lin,

Fudan University, China

Fabiana Melo,

Santa Casa de São Paulo, Brazi

*Correspondence:

Zhongqi Wang

Ihyyzlekzr07@hotmail.com

Specialty section

This article was submitted to Cancer Epidemiology and Prevention, a section of the journal

Frontiers in Oncology

Received: 20 July 2018 Accepted: 11 October 2018 Published: 31 October 2018

Citation:

Yang J, Li H, Deng H and Wang Z (2018) Association of One-Carbon Metabolism-Related Vitamins (Folate, B6, B12), Homocysteine and Methionine With the Risk of Lung Cancer: Systematic Review and Meta-Analysis. Front. Oncol. 8:493. doi: 10.3389/fonc.2018.00493

\section{Association of One-Carbon Metabolism-Related Vitamins (Folate, B6, B12), Homocysteine and Methionine With the Risk of Lung Cancer: Systematic Review and Meta-Analysis}

\author{
Jia Yang ${ }^{1}$, Hongjia $L^{2}{ }^{2}$, Haibin Deng ${ }^{1}$ and Zhongqi Wang ${ }^{1 *}$ \\ ' Oncology Department of LongHua Hospital, Shanghai University of Traditional Chinese Medicine, Shanghai, China, \\ ${ }^{2}$ Shanghai University of Traditional Chinese Medicine, Shanghai, China
}

Background: Studies on serum one-carbon metabolism factors (folate, B6, B12, homocysteine, and methionine) with lung cancer (LC) risk have produced inconsistent results. We aimed to systematically evaluate the association between them.

Methods: This study was reported in accordance with the PRISMA Statement and was registered with PROSPERO (no. CRD42018086654). Relevant studies were searched in PubMed, Embase, MEDLINE, and CNKI up to February 2018. Random-effects models were used to estimate the pooled standardized mean differences (SMD) or odds ratios (OR), as well as their 95\% confidence interval (Cl). Sensitivity and subgroup analysis were performed to identify the source of heterogeneity. Publication bias was also assessed.

Results: A total of 14 articles (8,097 patients) were included. The concentration of serum folate and vitamin B6 of LC patients were lower than the controls [SMD $-0.53,95 \%$ $\mathrm{Cl}(-0.70,-0.35), p=0.001$ and SMD $-0.28,95 \% \mathrm{Cl}(-0.53,-0.02), p=0.001$, respectively]. While the concentration of homocysteine of the cases was higher than the controls [SMD 0.41, 95\% Cl $(0.24,0.59), p=0.001]$. However, there were no significant differences between LC patients and the controls in terms of vitamin B12 and methionine [SMD $-0.09,95 \% \mathrm{Cl}(-0.27,0.09), p=0.202$ and SMD $-0.13,95 \% \mathrm{Cl}(-0.36,0.10)$, $p=0.001]$. Subgroup analysis showed that these results were more significant in Europe, Asia, former and current smokers, and the male population ( $p$-value $<0.05$ ).

Conclusions: Serum folate and vitamin B6 might be protective factors against lung carcinogenesis and homocysteine could contribute to LC risk.

Keywords: one-carbon metabolism, folate (vitamin B9), vitamin B6, lung cancer, serum, meta-analysis

\section{INTRODUCTION}

Despite advancement in treatment, lung cancer remains the most common and fatal cancer in the world (1). At present, $60 \%$ of lung cancer cases are in an advanced stage or metastatic at the time of diagnosis, highlighting the importance of finding serum biological variables in early stage of lung cancer (LC) (2). 
A growing body of evidence shows that DNA methylation plays an important role in the progression of cancers (3). DNA methylation requires a methyl donor, which is mainly provided by one-carbon metabolism (OCM) $(4,5)$. S-adenosylmethionine (SAM), the activated form of methionine, serves as the most important methyl donor. It can disrupt methionine cycle and change cytosine methylation in DNA by reducing intracellular SAM levels. In fact, the OCM involves a variety of factors, including folate (vitamin B9), vitamin B6, vitamin B12, homocysteine, and methionine. These factors interact with each other in a complex biochemical metabolic pathway. Through the mediating effects of these factors, one-carbon groups are transferred to maintain DNA methylation, regulate gene synthesis, and provide important material basis for biological functions $(6,7)$. Moreover, OCM is a dynamic process, in which the decrease or accumulation of one component can affect DNA methylation and genomic integrity, resulting in altered epigenetic modification, imbalanced of tumor suppressor genes and oncogene and induced malignant transformation (8).

However, researchers are still debating on the role of these factors in the development of LC. Some studies found no association between some OCM related factors and LC risk (912). However, increasing numbers of evidence have shown that $B$ vitamins and methionine were correlated with LC risk, not only in dietary supplements $(13,14)$ but also in serum $(15-17)$.

To the best of our knowledge, previous meta-analyses have not analyzed the relationship between all OCM factors in serum and LC risk. Thus, this study aims to systematically and comprehensively estimate the association of B vitamins (folate, B6, B12), homocysteine and methionine with LC risk in order to evaluate their important roles in LC.

\section{METHODS}

This systematic review and meta-analysis was reported in accordance with the Preferred Reporting Items for Systematic Reviews and Meta-Analysis (PRISMA) Statement (additional file: Checklist S1) and was registered with PROSPERO (no. CRD42018086654).

\section{Search}

We systematically searched the PubMed, Embase, MEDLINE and CNKI (China National Knowledge Infrastructure) databases to identify relevant studies up to February 1, 2018. The search, which placed no restrictions on language or publication status, used the following search terms: (Lung Neoplasms [MeSH]) AND ((Vitamin B9 [MeSH] OR Folate [MeSH]) OR (Vitamin B6 [MeSH] OR Pyridoxal 5-Phosphate [MeSH]) OR (Vitamin B12 [MeSH] OR Cyanocobalamin [MeSH]) OR Homocysteine [MeSH] OR Methionine [MeSH]). We also reviewed the relevant references cited in the retrieved articles. The search strategy used for PubMed is listed in Appendix A (Supplementary Material).

\section{Selection Criteria}

Studies were included in the meta-analysis if they met the following criteria: (1) the study focused on the association between serum B vitamins (folate, B6, B12) or homocysteine or methionine involved in one-carbon metabolism and lung cancer risk; (2) the outcome was lung cancer incidence; (3) case-control study, cohort study, or randomized controlled trial (RCT); and (4) the variables were reported as means (M) and standard deviations (SD), or as odds ratio (OR) and 95\% confidence intervals (95\% CI).

The exclusion criteria were (1) studies on dietary intake or supplementation of these B vitamins, homocysteine and methionine; (2) overlapping articles or studies that involved overlapping data; (3) conference abstract or meta-analyses; and (4) studies lacking healthy controls. If the same data were presented in more than one study, we included the study with the largest number of cases or provided more detailed information.

\section{Data Extraction}

Two investigators independently extracted the following data: first author, publication year, region/country, methods, study design, sample size, age, gender, source of controls and smoking status. We also extracted means (M) and standard deviations (SD) for the serum levels of B vitamins (folate, B6, B12), homocysteine and methionine as well as odds ratio (OR) and 95\% confidence interval (CI). To ensure the accuracy of the data, inconsistencies were discussed with another reviewer until a consensus was reached.

\section{Quality Assessment}

The Newcastle-Ottawa Scale (NOS) (18) was used to assess the methodological quality of the included studies (Table S1). The NOS consists of 3 aspects: selection, comparability, and exposure in the primary study. The total score ranged from 0 to 9 stars. Studies with 6 or more stars were classified as high-quality studies; studies with fewer than 6 stars were considered to be of low quality (19).

\section{Data Analysis}

All reported $P$-values were 2 -sided; $P$-values $<0.05$ were considered statistically significant. Standardized mean difference (SMD) and 95\% confidence interval (95\% CI) were used to estimate the serum folate, B6, B12, homocysteine and methionine in the overall and subgroup (by region, method) population; Odds ratio (OR) and $95 \%$ CI were used to evaluate these factors in subgroup population (by gender, smoking status). If the pooled effect size SMD $<0$ or $\mathrm{OR}<1$, the factor was protective; otherwise it was a risk factor.

Heterogeneity was calculated by Q test $\left(P_{\text {het }}\right)$ and $I$-squared $\left(I^{2}\right)$ statistics test. If $P_{h e t}<0.1$ or $I^{2}>30 \%$, then there was significant heterogeneity. The fixed-effect model was used if there was no heterogeneity; otherwise, a random-effect model was applied. If significant heterogeneity existed, sensitivity and subgroup analysis was used to explore heterogeneity sources and studies with 95\%CI markedly deviated from the others or factors leading to $I^{2}$ largely decreased were primary originators.

Publication bias were assessed using funnel plots and Egger test when the number of studies for biological variable was more than ten (20). If $P<0.1$, publication bias exited and then a 
trim-and-fill method was implemented to estimate the effect of publication bias on the results (21). All statistical analyses were performed using STATA version 12.0 software (Stata Corp LP, College Station, TX, USA).

\section{RESULTS}

\section{Literature Search and Study Characteristics}

A total of 1,458 articles were retrieved from the literature search, and 1,107 articles were obtained after the removal of duplicates. After the titles and abstracts read, 765 articles were found unrelated to the serum factors with lung cancer and were excluded. Then excluded were 302 studies about oral supplements, 4 conference abstracts, and 5 meta-analyses; after this exclusion, 31 articles remained. After careful reading of the full texts of these articles, 17 articles were further excluded from the study because 12 provided no information on relationship between serum factors and LC risk, 2 only included male population $(22,23), 3$ were duplicate data $(16,24,25)$. Thus, 14 articles $(17,22,23,26-38)$ were finally included into this systematic review and meta-analysis; The studies included 8,097 lung cancer patients and 10,008 healthy controls (Figure 1). Four studies were from Europe, nine were from Asia, and one was conducted in Europe, Asia, and America. Eleven of the studies were case-control studies, and three were nested case-control studies. The main features of these 14 studies are shown in Table 1.

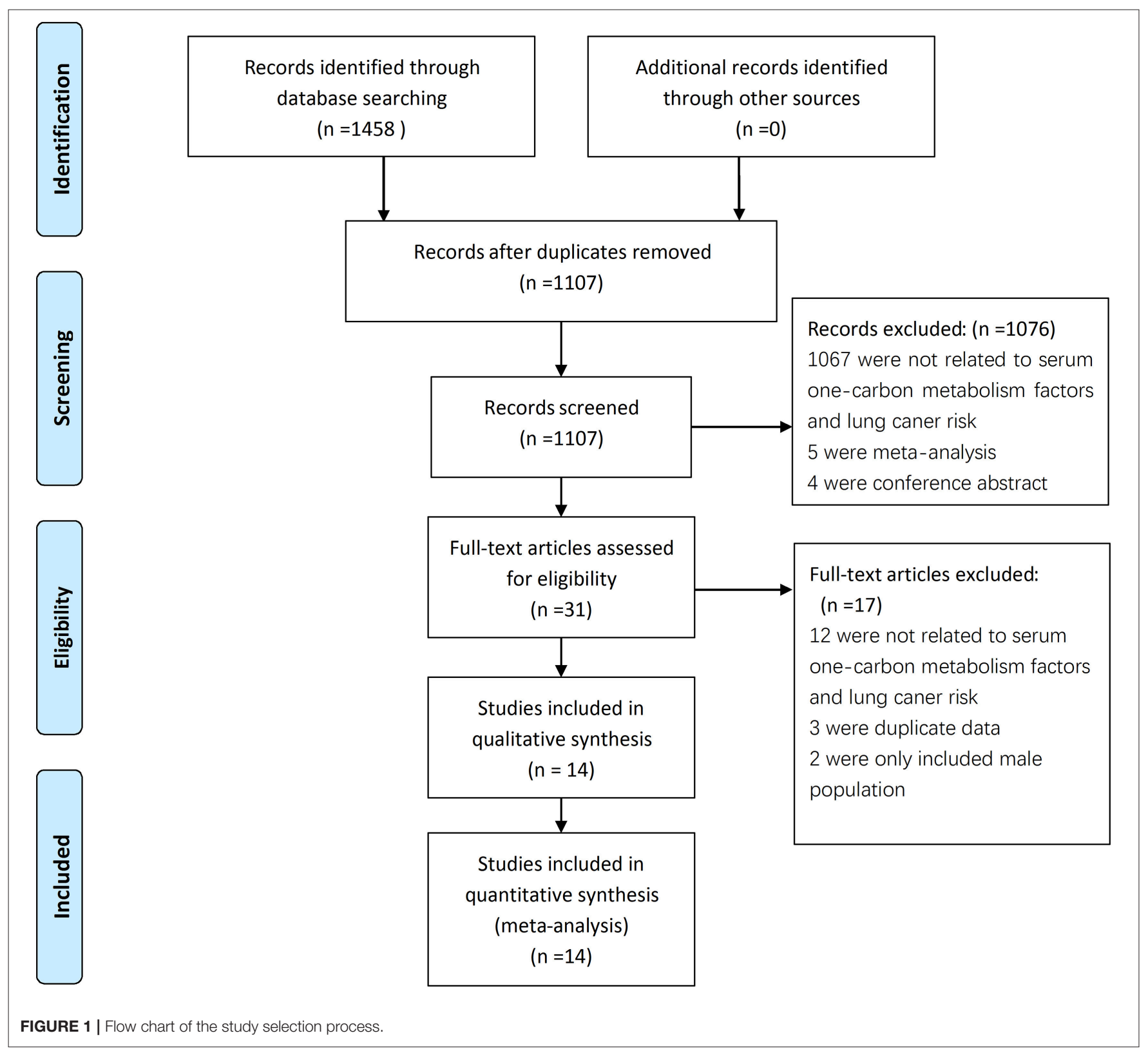




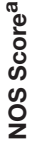

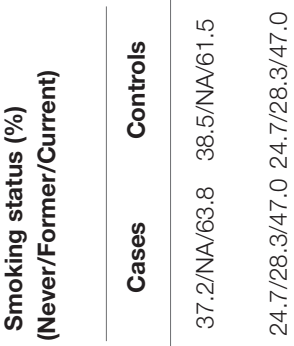

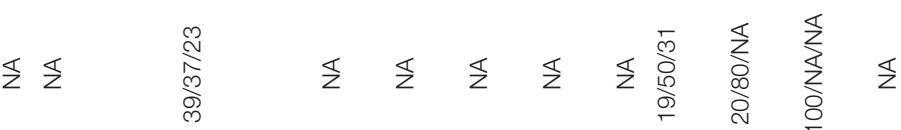

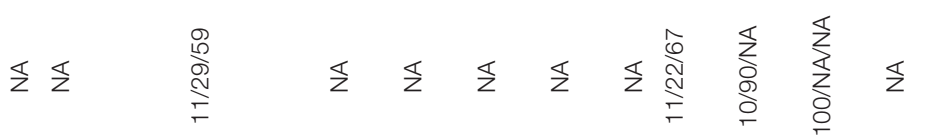

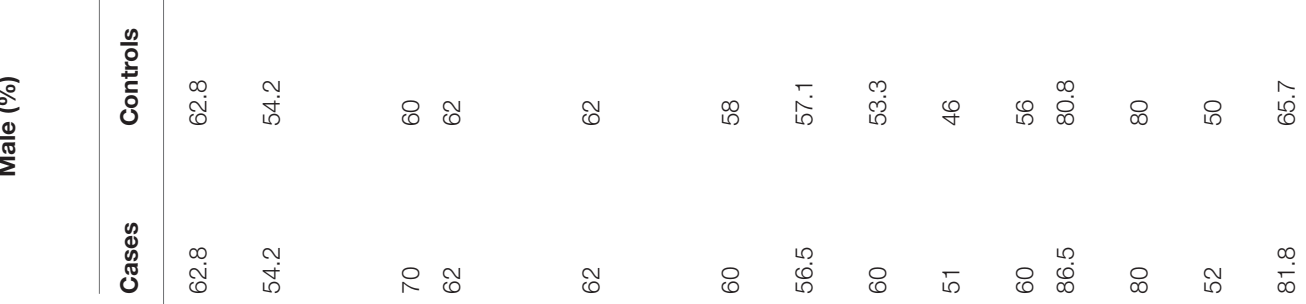

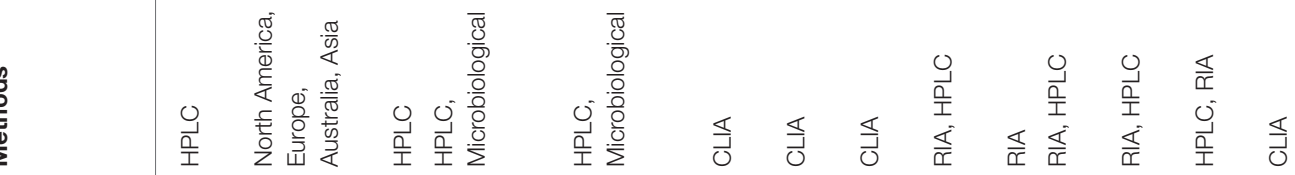

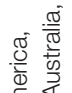

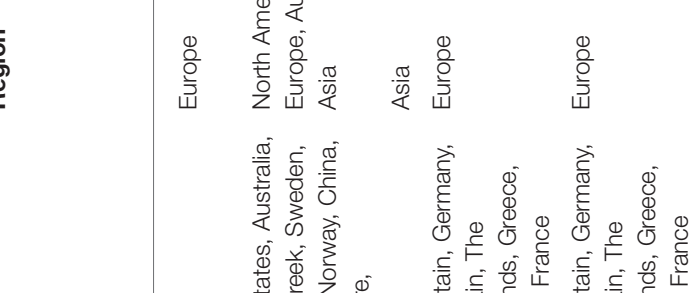

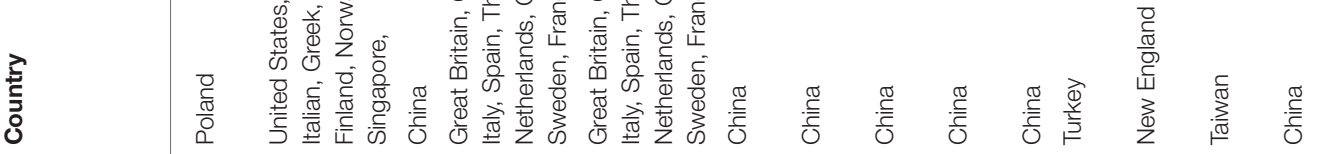

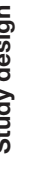


Yang et al.

Serum OCM and Lung Cancer

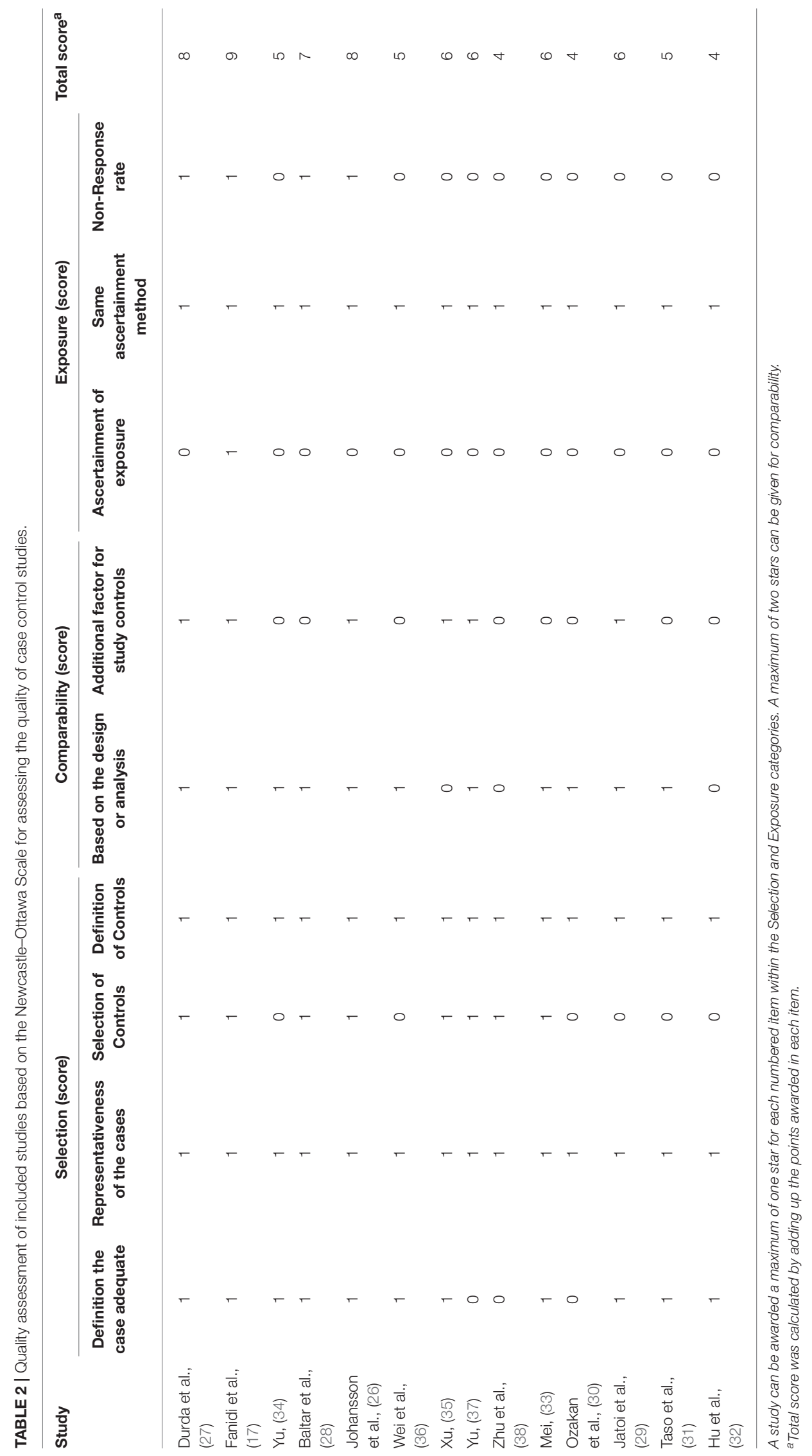

Frontiers in Oncology | www.frontiersin.org

October 2018 | Volume 8 | Article 493 


\section{Quality Assessment (the NOS Score)}

The result of quality assessment of the included studies based on Newcastle-Ottawa Scale (NOS) was summarized in Table 2. According to the quality evaluation criteria, these 14 studies scored from 4 to 9, and Eight of them were high-scoring studies (quality score $\geq 6$ ).

\section{Meta-Analysis}

Folate (Vitamin B9)

The concentration of serum folate of LC patients was significantly lower than the controls in the overall population, as shown in Figure 2 [SMD $-0.53,95 \%$ CI $(-0.70,-0.35), p=0.001]$. Further subgroup analyses were carried out according to clinical features. By region, serum folate level of LC patients was lower

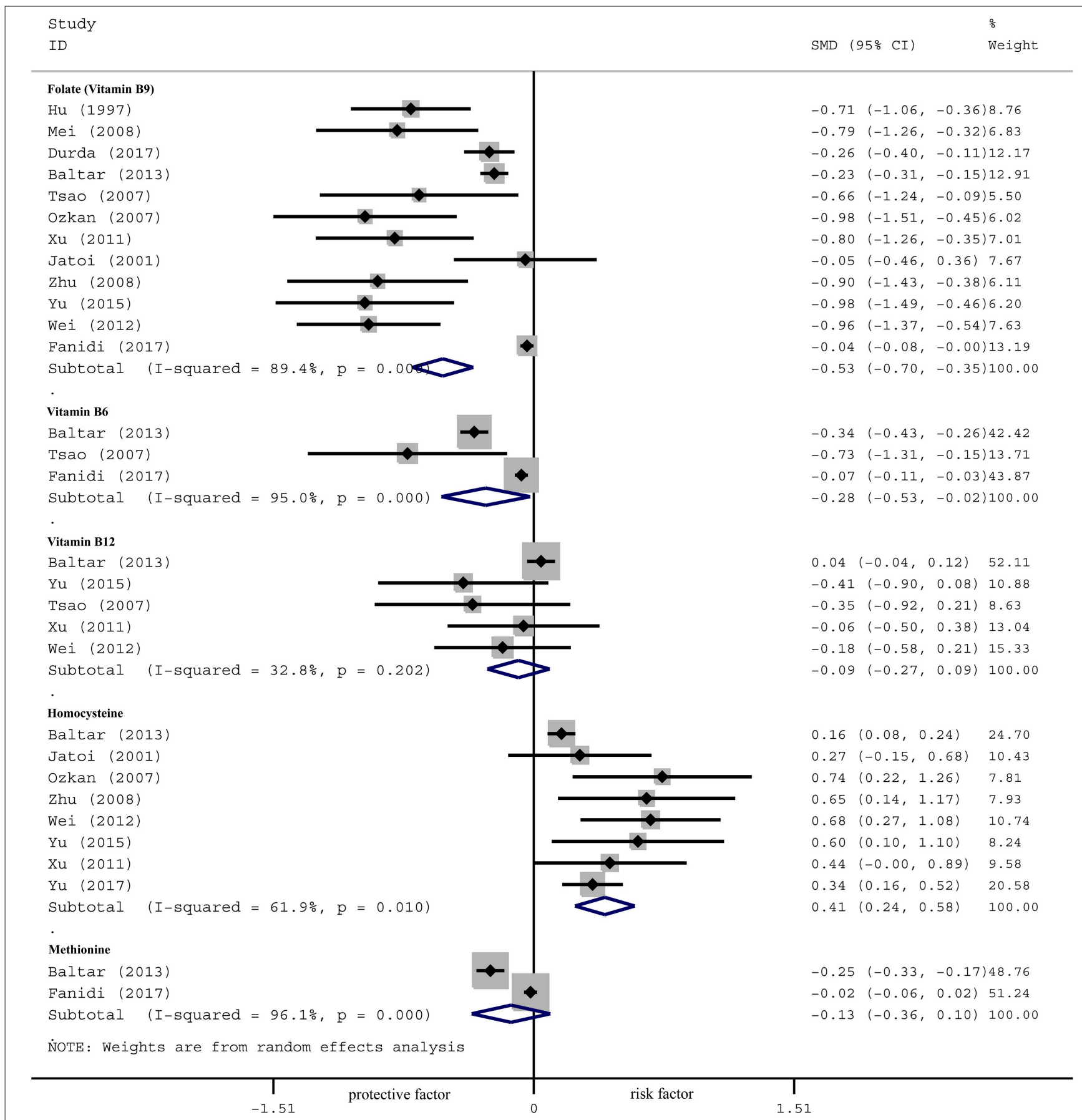

FIGURE 2 | Forest plot for OCM-related factors (folate, vitamin B6, vitamin B12, homocysteine, and methionine) and the risk of lung cancer. 
in Europe [SMD $-0.23,95 \%$ CI $(-0.30,-0.16), p=0.001]$ and Asia [SMD $-0.84,95 \% \mathrm{CI}(-1.01,-0.67), p=0.001]$, but not in the US [SMD $-0.02,95 \% \mathrm{CI}(-0.08,0.03), p=0.435]$. By method, serum folate level was lower in using CLIA/RIA [SMD $-0.74,95 \% \mathrm{CI}(-0.95,-0.54), p=0.001]$ and HPLC method [SMD -0.26 , 95\%CI $(-0.40,-0.11), p=0.001]$, but not by microbiological method [SMD -0.13 , 95\%CI $(-0.32$, $0.06), p=0.168$ ]. By gender, serum folate level was lower in male patients [OR 0.82, 95\%CI $(0.73,0.92), p=0.001$ ], but not in females [OR 0.94, 95\%CI $(0.84,1.05), p=0.272$ ]. By smoking status, the difference was especially significant in former smokers [OR $0.70,95 \% \mathrm{CI}(0.62,0.79), p=0.001$ ] and current smokers [OR 0.86, 95\%CI $(0.75,0.99), p=0.030]$, but was not significant in never smokers [OR 0.86 , 95\%CI $(0.75,1.0)$, $p=0.052]$ (Table 3).

\section{Vitamin B6}

The effect of serum B6 is presented in Figure 2 [SMD -0.28 , $95 \%$ CI $(-0.53,-0.02), p=0.001]$. Subgroup analysis by region showed that the concentration of serum B6 of LC patients was lower than the controls in Europe [SMD $-0.32,95 \% \mathrm{CI}(-0.41$, $-0.23), p=0.001$ ] and Asia [SMD $-0.38,95 \% \mathrm{CI}(-0.54,-0.23)$, $p=0.001]$, but not in the US [SMD $-0.04,95 \%$ CI $(-0.09$, $0.02), p=0.207]$. By gender, serum B6 level was lower in male patients than controls [OR $0.70,95 \% \mathrm{CI}(0.56,0.87), p=0.002$ ], but not in females [OR 1.08, 95\%CI $(0.98,1.21), p=0.152$ ]. By smoking status, the difference was especially significant in former smokers [OR $0.68,95 \% \mathrm{CI}(0.59,0.78), p=0.001$ ] and current smokers [OR $0.76,95 \% \mathrm{CI}(0.62,0.95), p=0.013$ ], but was not significant in never smokers [OR $0.95,95 \% \mathrm{CI}(0.36,2.48)$, $p=0.910]$ (Table 3).

\section{Vitamin B12}

No significant correlation was observed between serum B12 levels and lung cancer in the overall population [SMD -0.09 , $95 \%$ CI $(-0.27,0.09), p=0.202$ ] (Figure 2). Subgroup analysis showed no correlation in the Europe [SMD 0.04, 95\% CI $(-0.04$, $0.12), p=0.304$ ] nor Asia [SMD $-0.23,95 \%$ CI $(-0.46,0.01)$, $p=0.055]$. We did not observe association by method or smoking status (Table 3).

\section{Homocysteine (Hcy)}

Serum Hcy level of LC patients was higher than the controls in the overall population [SMD $0.41,95 \% \mathrm{CI}(0.24,0.58), p=0.001]$ (Figure 2). Further analysis by region showed that serum Hcy was significantly different between LC patients and the controls in both Europe [SMD 0.16, 95\% CI $(0.09,0.24), p=0.001]$ and Asia [SMD 0.45, 95\% CI (0.32, 0.59), $p=0.001$ ]. By method, serum Hcy was higher in patients than controls by using CLIA/RIA [SMD 0.58, 95\%CI $(0.32,0.84), p=0.001]$ and HPLC [SMD $0.34,95 \% \mathrm{CI}(0.15,0.52), p=0.001]$ method. No significant association was observed by smoking status (Table 3 ).

\section{Methionine}

No significant association between serum methionine levels and the risk of lung cancer was observed in the overall population [SMD $-0.13,95 \%$ CI $(-0.36,0.10), p=0.001$ ] (Figure 2). Further subgroup analysis by region showed that serum methionine was significantly different between LC patients and the controls in Europe [SMD -0.25, 95\%CI $(-0.33,-0.17)$, $p=0.027$ ], but not in Asia [SMD $-0.01,95 \%$ CI $(-0.07,0.06)$, $p=0.853$ ] nor the US [SMD -0.03 , 95\%CI $(-0.08,0.03)$, $p=0.395]$. No association was observed by subgroup analysis according to gender or smoking status (Table 3 ).

\section{Heterogeneity and Sensitivity Analysis}

Heterogeneity analysis showed significant heterogeneity in folate $\left(I^{2} 89.4 \%, P_{h e t} 0.061\right)$, B6 (I $\left.I^{2} 95.0 \%, P_{h e t} 0.039\right)$, B12 ( $I^{2} 32.8 \%$, $\left.P_{h e t} 0.015\right)$, Hcy $\left(I^{2} 61.9 \%, P_{h e t} 0.030\right)$ and methionine $\left(I^{2}\right.$ $96.1 \%, P_{\text {het }} 0.026$; Figure 2). We therefore performed a sensitivity analysis to identify the source of the heterogeneity (Figures S1S5). The results showed that the main clinical feature of the three studies (27-29) with markedly deviated 95\%CI were region, thus we firstly performed a subgroup analysis according to region. Meanwhile, considering that other clinical features could influence outcome, we also performed subgroup analyses based on the strata of method, gender and smoking status. Our results indicated that region was the primary heterogeneity originator in folate, vitamin B6, vitamin B12 and Hcy, as well as method in vitamin B12, gender in folate and smoking status in folate (Table 3).

\section{Publication Bias}

We assessed publication bias on the articles of serum folate and LC risk by funnel plot and Egger test (Figure 3), which suggested potential publication bias (Egger's test $p=0.038$ ). Then the trim-and-fill analysis was used to estimate the effect of publication bias. It was found that the results from random and fixed effects models were consistent with the original results (Table S2), indicating that there was no effect of these bias on the results, and this meta-analysis was reliable.

\section{DISCUSSION}

Among the factors associated with one-carbon metabolism, serum folate and vitamin B6 were found to be protective against lung cancer. Hcy was a risk factor for lung cancer. No significant correlation between $\mathrm{B} 12$ or methionine and the LC risk was observed.

\section{Association Between Serum OCM-Related Factors and LC Risk}

Folate acts as the core methyl donor in one-carbon metabolism, our results proved serum folate was a potential protective factor against lung carcinogenesis which was consistent with the results of other types of cancers, such as colorectal cancer (39), prostate cancer (40), and esophageal cancer (41). However, unlike a previous meta-analysis (15), our study showed statistically significant association between serum folate and the LC risk. This discrepancy may be explained by Dai study (15) included a cohort study $(n=15)$ (42) with insufficient cases and 4 casecontrol studies with inconsistent baseline. Although cohort study provided better evidence of causation than case-control study, the strength may be greatly reduced when the sample size is $<30$ with a high loss of follow-up rate. 


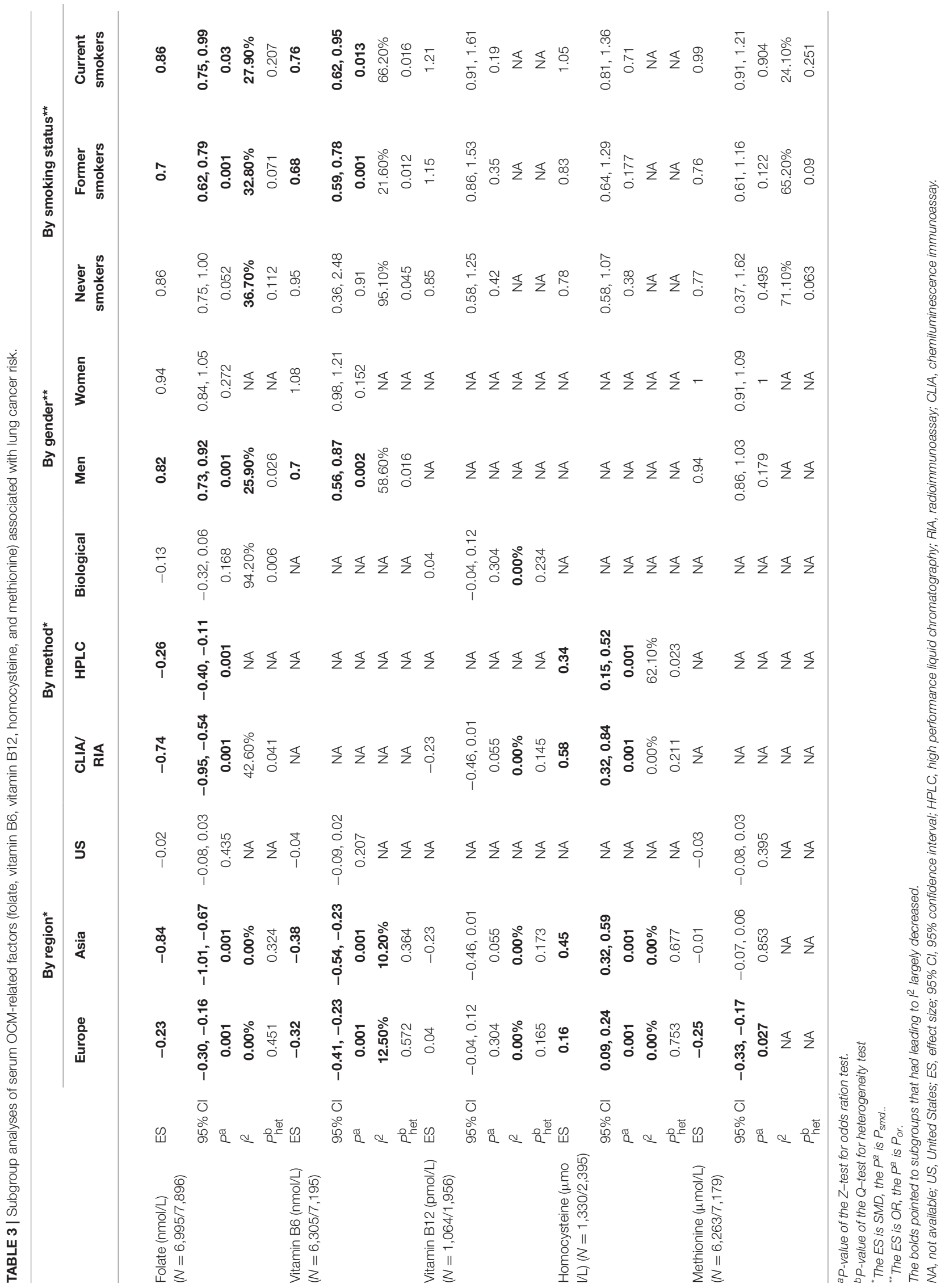


In addition to folate, we comprehensively analyzed the association between other important factors involved in the OCM and LC risk $(5,7)$. For vitamin B6, we considered it as a protective factor for lung carcinogenesis and similar results were discovered in gastrointestinal cancer (43), pancreatic cancer (44), and breast cancer (45). However, a prospective cohort study (HUSK) did not find any association between serum vitamin B6 and lung cancer risk (46). We speculated that the inconsistency of these results may due to the detection of different activated forms of vitamin B6 in blood. The HUSK used 4-pyridoxic acid/pyridoxal ratio (PAr) as a new form instead of classical pyridoxal 5' -phosphate (PLP), which may be instable and has low sensitivity to detect a significant difference.

In terms of Hcy, which acts as an essential intermediate in methionine metabolic cycle. Our findings suggest that serum Hcy could contribute to lung cancer and similar results were reported in all types of cancer in a previous meta-analysis (47). However, some studies did not observe the significant association $(22,26)$, which may be explained by these studies restricted to the older smoking men whose physical decline (or the accumulation of unhealthy habits) belying the role of Hcy.

Regarding vitamin B12 and methionine, we did not find an associated with LC risk. For vitamin B12, some studies argued it was associated with higher mortality of LC cases $(36,48)$. It is possible that vitamin B12 plays different roles in pathogenesis and prognosis. As to methionine, our findings were consistent with the LC3 study (17), but the subgroup analysis (only from the EPCI study) (26) showed that serum methionine were inversely associated with LC risk, which draws speculation that methionine protects Europeans from LC rather than other populations.

Particularly, subgroup analysis results showed that the significant association between folate, vitamin B6, Hcy and LC risk was detected in Asia and Europe, but not in the US. The null result may due to only a small number of studies from the US were included $(17,26)$. Thus, further researches from more regions would be needed. Moreover, it was speculated that the mediating effects of OCM factors of LC would change in different regions $(13,49)$. Secondly, folate and vitamin B6 were found protective in ever smokers (former and current smokers) but not significant in never smokers, which might be related to the nitrites in tobacco that could obstruct the function of $\mathrm{B}$ vitamins or decrease its blood values $(23,27,50,51)$. Thirdly, the significant association of folate and vitamin B6 with LC was in males indicates the function of regulating androgen signaling by the key enzymes of one-carbon metabolism $(9,14)$. Lastly, after subgroup analysis by different detecting methods, we found no difference of the association.

\section{Strengths and Limitations}

This is the first comprehensive and systematic evaluation of the relationship between serum folate, vitamin B6, vitamin B12, homocysteine, and methionine (OCM-related factors) and the LC risk. Previous meta-analyses focused only on the association of one or two OCM factors with LC or all types of cancer risk. Moreover, we investigated blood values instead of intake level, which can better reflect the association after the factors absorbed and metabolized in the body. In addition, this study included more than 8,000 lung cancer patients who participated

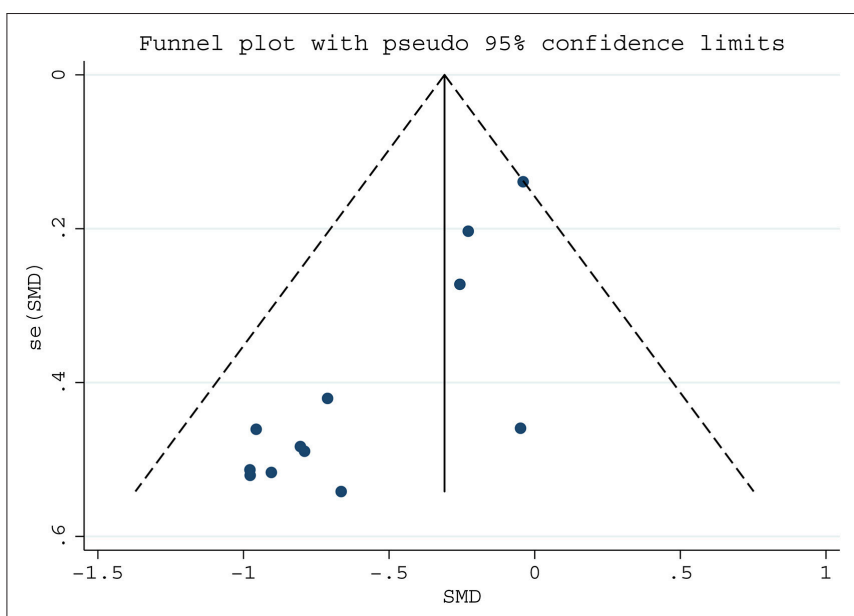

FIGURE 3 | Funnel plot for assessing publication bias for serum folate and lung cancer risk.

in very recent studies and analyzed by different subgroups, which reduced selection bias and corrected some errors in previous meta-analysis (15).

There were some limitations as well. First, although most of the original studies adjusted for variables, such as BMI, education, alcohol intake, tobacco exposure, and sex and we assessed potential sources of heterogeneity (region, gender, smoking status and method) through sensitivity analysis and subgroup analyses, we cannot completely rule out possible effects of unknown factors, such as pathology and clinical stages. Second, some epidemiological studies have shown that OCM related factors are potentially interrelated $(30,37)$, so we are expected to further analyze the synergistic effects of two or more factors or the dose-response on LC risk based on more data. Due to the lack of available cohort studies and RCT studies, we only included case-control studies and nested case-control studies. Eleven case-control studies might provide less certain evidence of causation, but there were three nested case-control studies with large sample size which provided more reliable evidence. The included studies were well-suited for meta-analysis as indicated by quality assessment, especially eight were high-scoring studies.

\section{CONCLUSIONS}

This study showed that among OCM-related factors, serum folate and vitamin B6 could function as protective factors against lung carcinogenesis but that serum homocysteine could contribute to lung cancer risk. No significant association was observed between vitamin $\mathrm{B} 12$ or methionine and $\mathrm{LC}$ risk. Our findings were significant in Europe and Asia, former and current smokers, and males. Further research is needed to validate these results in other populations.

\section{AUTHOR CONTRIBUTIONS}

JY and HL conceived and designed the study. JY, HL, and $\mathrm{HD}$ took full responsibility for data collecting and 
accuracy. JY and HL performed the meta-analysis, systematic review and drafted the manuscript. ZW helped revise the manuscript.

\section{FUNDING}

This work was supported by the National Natural Science Foundation of China (NSFC, No: 81703855) and the Project

\section{REFERENCES}

1. McKay JD, Hung RJ, Han Y, Zong X, Carreras-Torres R, Christiani DC, et al. Large-scale association analysis identifies new lung cancer susceptibility loci and heterogeneity in genetic susceptibility across histological subtypes. Nat Genet. (2017) 49:1126-32. doi: 10.1038/ng.3892

2. Mokdad AH, Dwyer-Lindgren L, Fitzmaurice C, Stubbs RW, BertozziVilla A, Morozoff C, et al. Trends and patterns of disparities in cancer mortality among US counties, 1980-2014. JAMA (2017) 317:388-406. doi: 10.1001/jama.2016.20324

3. Zhang Z, He Y, Tu X, Huang S, Chen Z, Wang L, et al. Mapping of DNA hypermethylation and hypomethylation induced by folate deficiency in sporadic colorectal cancer and clinical implication analysis of hypermethylation pattern in CBS promoter. Clin Lab. (2017) 63:733-48. doi: 10.7754/Clin.Lab.2016.161018

4. Kurzius-Spencer M, da Silva V, Thomson CA, Hartz V, Hsu CH, Burgess JL, et al. Nutrients in one-carbon metabolism and urinary arsenic methylation in the National Health and Nutrition Examination Survey (NHANES) 2003-2004. Sci Total Environ. (2017) 607-608:381-90. doi: 10.1016/j.scitotenv.2017.07.019

5. Newman AC, Maddocks ODK. One-carbon metabolism in cancer. Br J Cancer (2017) 116:1499-504. doi: 10.1038/bjc.2017.118

6. Shuvalov O, Petukhov A, Daks A, Fedorova O, Vasileva E, Barlev NA. One-carbon metabolism and nucleotide biosynthesis as attractive targets for anticancer therapy. Oncotarget (2017) 8:23955-77. doi: 10.18632/oncotarget.15053

7. Midttun $\varnothing$, Theofylaktopoulou D, McCann A, Fanidi A, Muller DC, Meyer $\mathrm{K}$, et al. Circulating concentrations of biomarkers and metabolites related to vitamin status, one-carbon and the kynurenine pathways in US, Nordic, Asian, and Australian populations. Am J Clin Nutr. (2017) 105:1314-26. doi: 10.3945/ajcn.116.151241

8. Ham MS, Lee JK, Kim KC. S-adenosyl methionine specifically protects the anticancer effect of 5-FU via DNMTs expression in human A549 lung cancer cells. Mol Clin Oncol. (2013) 1:373-78. doi: 10.3892/mco.2012.53

9. Brasky TM, White E, Chen CL. Long-term, supplemental, one-carbon metabolism-related vitamin B use in relation to lung cancer risk in the vitamins and lifestyle (VITAL) cohort. J Clin Oncol. (2017) 35:3440-8. doi: 10.1200/JCO.2017.72.7735

10. Cho E, Hunter DJ, Spiegelman D, Albanes D, Beeson WL, van den Brandt $\mathrm{PA}$, et al. Intakes of vitamins $\mathrm{A}, \mathrm{C}$ and $\mathrm{E}$ and folate and multivitamins and lung cancer: a pooled analysis of 8 prospective studies. Int J Cancer (2006) 118:970-8. doi: 10.1002/ijc.21441

11. Qin X, Cui Y, Shen L, Sun N, Zhang Y, Li J, et al. Folic acid supplementation and cancer risk: a meta-analysis of randomized controlled trials. Int J Cancer (2013) 133:1033-41. doi: 10.1002/ijc.28038

12. Vollset SE, Clarke R, Lewington S, Ebbing M, Halsey J, Lonn E, et al. Effects of folic acid supplementation on overall and site-specific cancer incidence during the randomised trials: meta-analyses of data on 50,000 individuals. Lancet (2013) 381:1029-36. doi: 10.1016/S0140-6736(12)62001-7

13. Ebbing M, Bønaa KH, Nygård O, Arnesen E, Ueland PM, Nordrehaug JE, et al. Cancer incidence and mortality after treatment with folic acid and vitamin B12. JAMA (2009) 302:2119-26. doi: 10.1001/jama.2009.1622

14. Takata Y, Cai Q, Beeghly-Fadiel A, Li H, Shrubsole MJ, Ji BT, et al. Dietary $\mathrm{B}$ vitamin and methionine intakes and lung cancer risk among of Scientific Research and Innovation of Shanghai Education Committee (No: 2017-01-07-00-10-E00064).

\section{SUPPLEMENTARY MATERIAL}

The Supplementary Material for this article can be found online at: https://www.frontiersin.org/articles/10.3389/fonc. 2018.00493/full\#supplementary-material

female never smokers in China. Cancer Causes Control (2012) 23:1965-75. doi: 10.1007/s10552-012-0074-Z

15. Dai WM, Yang B, Chu XY, Wang YQ, Zhao $M$, Chen L, et al. Association between folate intake, serum folate levels and the risk of lung cancer: a systematic review and meta-analysis. Chin Med J. (2013) 126:1957-64. doi: 10.3760/cma.j.issn.0366-6999.20130391

16. Chuang SC, Fanidi A, Ueland PM, Relton C, Midttun O, Vollset SE, et al. Circulating biomarkers of tryptophan and the kynurenine pathway and lung cancer risk. Cancer Epidemiol Biomarkers Prev. (2014) 23:461-8. doi: 10.1158/1055-9965.EPI-13-0770

17. Fanidi A, Muller DC, Yuan JM, Stevens VL, Weinstein SJ, Albanes D, et al. Circulating folate, Vitamin B6, and methionine in relation to lung cancer risk in the lung cancer cohort consortium (LC3). J Natl Cancer Inst. (2017) 110:57-7. doi: 10.1093/jnci/djx119

18. Cota GF, de Sousa MR, Fereguetti TO, Rabello A. The Newcastle-Ottawa Scale (NOS) for Assessing the Quality of Nonrandomized Studies in MetaAnalysis (2011). Available online at: http://www.ohri.ca/programs/clinical_ epidemiology/oxford.asp (Accessed November 25, 2012).

19. Higgins JP, Green S. Cochrane Handbook for Systematic Reviews of Interventions, Version 5.1 .0 (2011). Available online at: https://training. cochrane.org/handbook

20. Sterne JA, Gavaghan D, Egger M. Publication and related bias in metaanalysis: power of statistical tests and prevalence in the literature. J Clin Epidemiol. (2000) 53:1119-29. doi: 10.1016/S0895-4356(00)00242-0

21. Duval S, Tweedie R. Trim and fill: a simple funnel-plot-based method of testing and adjusting for publication bias in meta-analysis. Biometrics (2000) 56:455-63. doi: 10.1111/j.0006-341X.2000.00455.x

22. Hartman TJ, Woodson K, Stolzenberg-Solomon R, Virtamo J, Selhub J, Barrett MJ, et al. Association of the B-vitamins pyridoxal 5' -phosphate (B6), B12, and folate with lung cancer risk in older men. Am J Epidemiol. (2001) 153:688-94. doi: 10.1093/aje/153.7.688

23. Tastekin D, Erturk K, Bozbey HU, Olmuscelik O, Kiziltan H, Tuna S, et al. Plasma homocysteine, folate and vitamin B12 levels in patients with lung cancer. Exp Oncol. (2015) 37:218-22.

24. Theofylaktopoulou D, Midttun $\varnothing$, Ueland PM, Meyer K, Fanidi A, Zheng W, et al. Impaired functional vitamin B6 status is associated with increased risk of lung cancer. Int J Cancer (2017) 142:2425-34. doi: 10.1002/ijc.31215

25. Zuo H, Ueland PM, Midttun $\varnothing$, Vollset SE, Tell GS, Theofylaktopoulou D, et al. Results from the European prospective investigation into cancer and nutrition link Vitamin B6 catabolism and lung cancer risk. Cancer Res. (2018) 78:302-8. doi: 10.1158/0008-5472.CAN-17-1923

26. Johansson M, Relton C, Ueland PM, Vollset SE, Midttun $\varnothing$, Nygård O, et al. Serum B vitamin levels and risk of lung cancer. JAMA (2010) 303:2377-85. doi: 10.1001/jama.2010.808

27. Durda K, Kaklewski K, Gupta S, Szydłowski M, Baszuk P, Jaworska-Bieniek K, et al. Serum folate concentration and the incidence of lung cancer. PLoS ONE (2017) 12:e0177441. doi: 10.1371/journal.pone.0177441

28. Baltar VT, Xun WW, Johansson M, Ferrari P, Chuang SC, Relton C, et al. A structural equation modelling approach to explore the role of B vitamins and immune markers in lung cancer risk. Eur J Epidemiol. (2013) 28:677-88. doi: $10.1007 /$ s10654-013-9793-Z

29. Jatoi A, Daly DB, Kramer G, Mason JB. Folate status among patients with nonsmall cell lung cancer: a case-control study. J Surg Oncol. (2001) 77:247-52. doi: 10.1002/jso. 1104 
30. Ozkan Y, Yardim-Akaydin S, Firat H, Calişkan-Can E, Ardiç S, Simşek B. Usefulness of homocysteine as a cancer marker: total thiol compounds and folate levels in untreated lung cancer patients. Anticancer Res. (2007) 27:1185-9.

31. Tsao SM, Yin MC, Liu WH. Oxidant stress and B vitamins status in patients with non-small cell lung cancer. Nutr Cancer (2007) 59:8-13. doi: 10.1080/01635580701365043

32. Hu J, Zhu Y, Lu Y. The clinical signif icance of measuring the serum folic acid levels of patients with the lung cancer. Acta Universitatis Medicinalis Anhui (1997) 32:113-4.

33. Mei Y. Detection of serum folate in patients with malignant tumor. Gansu Med J. (2008) 27:78-9. doi: 10.15975/j.cnki.gsyy.2008.04.046

34. Yu J. Analysis of abnormal blood lipid and Homocysteine levels in patients with different malignant tumors. Guangxi Med Univ. (2017) 07:579-561. doi: 10.14163/j.cnki.11-5547/r.2017.04.011

35. $\mathrm{Xu} \mathrm{C}$. Clinical significance of determination of serum homocysteine in patients with lung cancer. J Chin Phys. (2011) 13:533-4. doi: 10.3760/cma.j.issn.1008-1372.2011.04.039

36. Wei K, Huang Y. Relationship between plasma homocysteine, folate and vitamin B12 levels and non-small cell lung cancer. Int J Lab Med. (2012) 33:699-701. doi: 10.3969/j.issn.1673-4130.2012.06.028

37. Yu X, Wang W, Wu X, Liu X. Detection of serum folate, homocysteine and vitamin $\mathrm{B} 12$ in patients with non-small cell lung cancer and its clinical significance. J Jilin Univ. (2015). 41:1235-1238. doi: 10.13481/j.1671-587x.20150626

38. Zhu F, Zhang Q, Wang Y. Plasma homocysteine and serum folic acid levels in patients with malignant tumor. Suzhou Univ J Med Sci. (2008) 28:1041-42. doi: 10.3969/j.issn.1002-266X.2008.31.064

39. Takata Y, Shrubsole MJ, Li H, Cai Q, Gao J, Wagner C, et al. Plasma folate concentrations and colorectal cancer risk: a case-control study nested within the Shanghai Men's Health Study. Int J Cancer (2014) 135:2191-8. doi: $10.1002 /$ ijc. 28871

40. de Vogel S, Meyer K, Fredriksen Å, Ulvik A, Ueland PM, Nygård O., et al. Serum folate and vitamin B12 concentrations in relation to prostate cancer risk-a Norwegian population-based nested case-control study of 3000 cases and 3000 controls within the JANUS cohort. Int J Epidemiol. (2013) 42:20110. doi: 10.1093/ije/dys199

41. Zhao Y, Guo $\mathrm{C}$, Hu H, Zheng L, Ma J, Jiang L, et al. Folate intake, serum folate levels and esophageal cancer risk: an overall and dose-response meta-analysis. Oncotarget (2017) 8:10458-69. doi: 10.18632/oncotarget. 14432

42. Rossi E, Hung J, Beilby JP, Knuiman MW, Divitini ML, Bartholomew H. Folate levels and cancer morbidity and mortality: prospective cohort study from Busselton, Western Australia. Ann Epidemiol. (2006) 16:206-12. doi: 10.1016/j.annepidem.2005.03.010
43. Gylling B, Myte R, Schneede J, Hallmans G, Häggström J, Johansson I, et al. Vitamin B-6 and colorectal cancer risk: a prospective population-based study using 3 distinct plasma markers of vitamin B-6 status. Am J Clin Nutr. (2017) 105:897-904. doi: 10.3945/ajcn.116.139337

44. Huang JY, Butler LM, Midttun $\varnothing$, Koh WP, Ueland PM, Wang R, et al. Serum B6 vitamers (pyridoxal 5 ' -phosphate, pyridoxal, and 4-pyridoxic acid) and pancreatic cancer risk: two nested case-control studies in Asian populations. Cancer Causes Control (2016) 27:1447-56. doi: 10.1007/s10552-016-0822-6

45. Wu W, Kang S, Zhang D. Association of vitamin B6, vitamin B12 and methionine with risk of breast cancer: a dose-response meta-analysis. $\mathrm{Br} \mathrm{J}$ Cancer (2013) 109:1926-44. doi: 10.1038/bjc.2013.438

46. Zuo H, Ueland PM, Eussen SJ, Tell GS, Vollset SE, Nygård O, et al. Markers of vitamin B6 status and metabolism as predictors of incident cancer: the Hordaland Health Study. Int J Cancer (2015) 136:2932-9. doi: 10.1002/ijc.29345

47. Zhang W, Braun A, Bauman Z, Olteanu H, Madzelan P, Banerjee R Expression profiling of homocysteine junction enzymes in the NCI60 panel of human cancer cell lines. Cancer Res. (2005) 65:1554-60. doi: 10.1158/0008-5472.CAN-04-1554

48. Zhou XY, Feng SX, Li XL, Liu YD, Chen X, Ye DS, et al. Elevated plasma vitamin B12 levels and cancer prognosis: a population-based cohort study. Cancer Epidemiol. (2016) 40:158-65. doi: 10.1016/j.canep.2015.12.007

49. Yang Q, Bostick RM, Friedman JM, Flanders WD. Serum folate and cancer mortality among U.S. adults: findings from the third national health and nutritional examination survey linked mortality file. Cancer Epidemiol Biomarkers Prev. (2009) 18:1439-47. doi: 10.1158/1055-9965.EPI-08-0908

50. Suzuki T, Matsuo K, Hiraki A, Saito T, Sato S, Yatabe Y, et al. Impact of one-carbon metabolism-related gene polymorphisms on risk of lung cancer in Japan: a case control study. Carcinogenesis (2007) 28:1718-25. doi: 10.1093/carcin/bgm104

51. Shi Q, Zhang Z, Li G, Pillow PC, Hernandez LM, Spitz MR, et al. Sex differences in risk of lung cancer associated with methylene-tetrahydrofolate reductase polymorphisms. Cancer Epidemiol Biomarkers Prev. (2005) 14:1477-84. doi: 10.1158/1055-9965.EPI-04-0905

Conflict of Interest Statement: The authors declare that the research was conducted in the absence of any commercial or financial relationships that could be construed as a potential conflict of interest.

Copyright (C) 2018 Yang, Li, Deng and Wang. This is an open-access article distributed under the terms of the Creative Commons Attribution License (CC BY). The use, distribution or reproduction in other forums is permitted, provided the original author(s) and the copyright owner(s) are credited and that the original publication in this journal is cited, in accordance with accepted academic practice. No use, distribution or reproduction is permitted which does not comply with these terms. 